\title{
PARALLEL AND ADAPTIVE UNIFORM-DISTRIBUTED REGISTRATION METHOD FOR CHANG'E-1 LUNAR REMOTE SENSED IMAGERY
}

\author{
X. Ning ${ }^{\mathrm{a}, *}, \mathrm{M} . \mathrm{Hao}^{\mathrm{a}}$, \\ ${ }^{a}$ Institute of Photogrammetry and Remote Sensing, Chinese Academy Surveying and Mapping, 28 Lianhuachi West \\ Road, Beijing, China - (ningxg, haomh)@ casm.ac.cn
}

\section{Commission VI, WG VI/4}

KEY WORDS: Chang'E-1, registration, SIFT, parallel calculation, uniform distribution

\begin{abstract}
:
On the October $24^{\text {th }} 2007$, China launched its first Lunar Probe Satellite "Chang'E I". After the 494 days travelling, the probe vehicle landed accurately at its predetermined landing site on the moon at 52.36 degrees east longitude and 1.5 degrees south latitude. It sent back the first imagery of the lunar surface on 26 November 2007 and accomplished all the scheduled scientific tasks successfully. As the first lunar Probe Satellite, the major goal of Chang'E I mission is to obtain three-dimensional images of the landforms and geological structures of the lunar surface, so as to provide a reference for planned future soft landings. However, due to the dramatic change of the radiation information of the CE-limagery, the traditional methods that are based on the gray and line characters show the limitation achieving a satisfied result. Moreover, the registration processing between lunar images that cover the whole moon has proved to be very time-consuming.

To resolve the above-mentioned registration difficulties, a parallel and adaptive uniform-distributed registration method for CE-1 lunar remote sensed imagery is proposed in this paper. Based on 6 pairs of randomly selected images, both the standard SIFT algorithm and the parallel and adaptive uniform-distributed registration method were executed, the versatility and effectiveness were assessed. The experimental results indicate that: by applying the parallel and adaptive uniform-distributed registration method, the efficiency of CE-1 lunar remote sensed imagery registration were increased dramatically. Therefore, the proposed method in the paper could acquire uniform-distributed registration results more effectively, the registration difficulties including difficult to obtain results, time-consuming, non-uniform distribution could be successfully solved.
\end{abstract}




\section{INTRUDUCTION}

On the October $24^{\text {th }} 2007$, China launched its first lunar Probe Satellite "Chang'E I". After the 494 days travelling, the probe vehicle landed at its predetermined landing site on the moon at 52.36 degrees east longitude and 1.5 degrees south latitude accurately (Li et al., 2010). It sent back the first imagery of the lunar surface on 26 November 2007 and accomplished all the scheduled scientific tasks successfully. As the first lunar Probe Satellite, the major goal of Chang'E I mission is to obtain threedimensional images of the landforms and geological structures of the lunar surface, so as to provide a reference for planned future soft landings (Zheng et al., 2007).

Due to the dramatic change of the radiation information of the CE-1imagery, the traditional gray and line characters based matching method has shown the limitation achieving a satisfied result. By analyzing the imaging principle and quality of the CE-1 satellite, the Scale Invariant Feature Transform (or SIFT) algorithm is chosen as the research-based algorithm in this paper. SIFT is an algorithm mainly applied in computer vision to detect and describe local features in images. The SIFT algorithm was published by David Lowe in 1999 (David G,1999) and then improved in 2004 (David G, 2004). One important characteristic of the SIFT algorithm is that its feature descriptor is invariant to uniform scaling, orientation, and partially invariant to affine distortion and illumination changes (Mikolajczyk and Schmid, 2005, Sun, 2005), therefore shows the suitability in image matching when great gray value differences exist. Both the traditional gray-based and the SIFT image matching algorithm are conducted for the CE-1 lunar imagery matching in this research, the experimental results indicate the efficiency and applicability of SIFT for feature extraction and matching (Liu, 2009, Liu 2010).

The rest of the paper in organized as follows. The imaging principle of the CCD stereo camera of CE-1 satellite and the core theory of the SIFT matching algorithm are presented first. To improve the efficiency of the matching operation and solve the uneven distributed matching points, the paralleled matching method is further studied in this paper and then a parallel and adaptive uniform-distributed registration method for Chang'e-1 lunar remote sensed imagery is proposed. Based on the 6 pairs of randomly selected images, the performance of the proposed strategy through the comparison of its experimental results with the results generating from the standard SIFT algorithm is evaluated. Finally, the conclusion part summarizes the work of the paper and gives a simple view of problems that need further study in this field.

\section{IMAGING PRINCIPLE OF CE-1 SATELLITE AND SIFT ALGORITHM}

\subsection{Imaging principle of CCD stereo camera on CE-1}

The CE-1 satellite is assembled with a three-liner-array CCD as the receiving device and designed with a orbit altitude of $200 \mathrm{~km}$. The three line-array CCD are used to collect the forward, nadir and backward looking of the imaging track separately while the off-nadir viewing across the track is $17^{\circ}$ (Wang et al., 2008, Zhao et al., 2009, ), see Figure 1(left). The CE-1 camera has the pixel size of $14 \mathrm{um}$ and is designed with a $23.33 \mathrm{~mm}$ 's focal length. In the camera imaging process, the arrays of 11, 512 and 1013 of the CCD matrix take ground features at the same time, as presented in Figure 1 (right). This scanning and imaging method determines the high overlapping characteristic of the stripes among the forward, nadir and backward view, which could be up to $96 \%$.
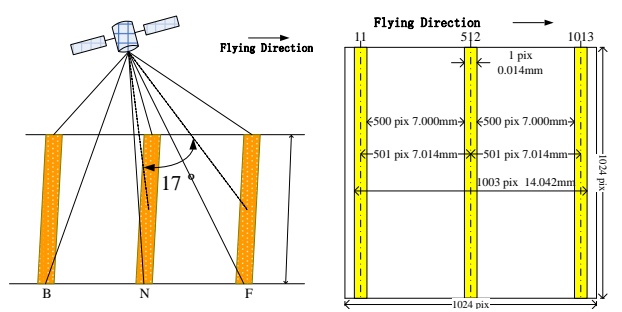

Figure 1 Imaging principal of CCD camera (left), imaging principal of CCD camera (right)

The radiation information of the CE-1 lunar imageries changes violently. The pictures below represent the radiation variation of the CE-1 images captured from different areas: (1) low radiation area, (2) high radiation area, and (3)(4) high radiation contrast in the continuous area. This phenomenon results in difficulties in image matching by using the traditional gray-based or the feature point and feature line characteristic based methods.

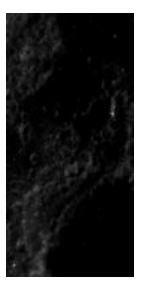

(1)

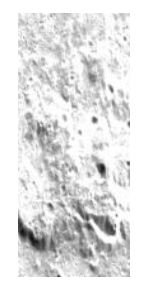

(2)

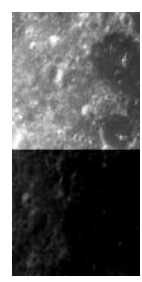

(3)

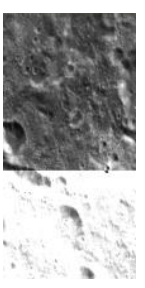

(4)
Figure 2 Comparison of radiation information from different areas on CE-1 images

\subsection{SIFT algorithm}

The scale invariant feature transform (SIFT) algorithm, was firstly proposed by Lowe in 1999 and then further developed in 2004 . The core ideology of SIFT algorithm is locating the local extrema at the scale space and further detecting the invariant image features. Compared with the feature-based algorithm, the SIFT is invariant to image translation, scaling, rotation and partially invariant to illumination changes and affine projection. SIFT image registration is conducted through the following five steps.

1) Generating Gaussian and DOG images at Scale-space. Gaussian kernel is the only linear kernel for scale transformation (Koenderink, 1984, Lindeberg, 1994). To build the DOG pyramid the input images are up-sampled and convolved iteratively with a Gaussian kernel of $\sigma=1.5$. After this step, 5 Gaussian pyramid are generated and each of them contains 5 layers, thus the Difference of Gaussians (DOG) pyramid can be computed by conducting the differential operation onto the two nearby images.

2) Extrema point location and marginal point elimination. The local extrema point is detected by comparing with its neighboring 8 points in the same scale level, 9 neighboring points in the scale above and scale below each. In the DOG processing, the local maximum or minimum is considered as the candidate till all the 26 neighboring points are assessed. The SIFT algorithm works by fitting a $3 \mathrm{D}$ quadratic function to define the location and the scale of the accurate key-points (reaching the sub-pixel accuracy), and discarding the lowcontrast such as the unstable edge-corresponding points. For the selected feature points, the Taylor expansion is conducted first to calculate the accurate location of the local extrema and then the edge-corresponding points are excluded though the Hessian matrix procedure. 
3) Orientation assignment for the feature points. Once the location of the feature points is determined, a buffering area is assigned to each feature then the gradient and the weight value of each pixel in the local neighborhood are calculated. An orientation histogram is established by the weighted gradient magnitudes: the highest orientation histogram peak represents the principal direction of the feature points while the peaks with amplitudes greater than $80 \%$ of the highest peak are used to create a assistant orientation with this point.

4) Sift-descriptor generation. Centered as the Sift feature point, selecting a region of $16 * 16$ as the variables windows and further dividing it into $4 * 4$ boxes. The gradient magnitudes and orientations within each $4 * 4$ box are computed and an 8 bins orientation histogram could be established. From the 16 obtained orientation histograms, a 128 dimensional vector (SIFT-descriptor) is built.

5) Sift feature matching. To determine the corresponding features between two images, the Euclidean distance between SIFT descriptors is introduced as the indicator to measure the similarity. Selecting one SIFT-descriptor from image A and its corresponding descriptors that represents the first two nearest Euclidean distance from image B. As proposed by Lowe, if the ratio of the Euclidean distance of the nearest and the second nearest neighbors is less than a certain threshold $\tau$, this pair of matching points is accepted. The total amount of matching points becomes smaller by reducing the threshold value, while the results appear more stable. In this paper, the threshold is set as 0.6 .

\section{PARALLEL AND ADAPTIVE UNIFORM- DISTRIBUTED REGISTRATION METHOD FOR CHANG'E-1 IMAGERY}

\subsection{Parallel registration method}

In consideration of scale factor, overlapping degree as well as the application requirements, the forward (F) and backward (B) view of the imaging track are selected in this research for CE-1 stereo image registration. As shown in Figure 1 in the section 2, the coverage discrepancy between $\mathrm{B}$ and $\mathrm{F}$ view images can be calculated as the following:

$$
S=2 \mathrm{H} \tan \varphi
$$

$\mathrm{H}$ is the flying height, represents the angle between different views. Based on the formula above, the calculated $\mathrm{S}$ is $122 \mathrm{~km}$ while corresponding to 1000 pixels. The overlapping area between the $\mathrm{F}$ and $\mathrm{B}$ views presents in the Figure 3.
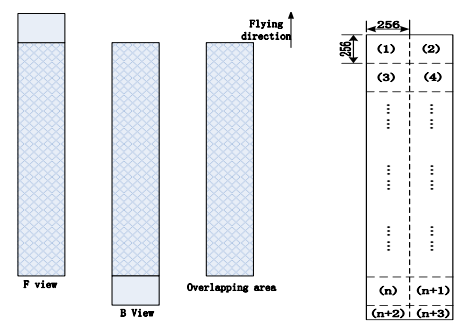

Figure 3 Overlapping area between B and F view (left), blocks clipped from overlapping area (right)

As has been described in section 2.2, the SIFT is a computeintensive algorithm, while the most intensive part focuses on the phase of Sift-descriptor generation (step1-4). In addition, the
SIFT-descriptor generation only requires partial images in the processing procedure. Due to the large image data size for the full-coverage lunar map generation, this method shows the feasibility for the image parallel processing.

Based on above analysis, the overlapping area between $\mathrm{B}$ and $\mathrm{F}$ view were introduced in this paper. To reduce the amount of image calculation, the overlapping area is sequentially partitioned into several blocks with a fixed size of $256 * 256$ pixels from left to right, from top to bottom (see Figure 3, right). For the marginal part of the overlapping area (less than 256*256 pixels), the original size will be kept and participated in the further operation in the later stage.

After the partition step, the SIFT descriptors are processed on the basis of each generated overlapping block parallelly to realize the full image's matching.

\subsection{Adaptive uniform-distributed registration method}

In practical SIFT algorithm application for the CE-1 lunar imagery registration; it has been found that the distribution of the matching points is too dense and not uniform. To avoid data redundancy, enhance accuracy and the processing efficiency, an adaptive uniform-distributed registration method is proposed in this paper.

The basic approach of the adaptive uniform-distributed registration method is: ranking the original Gaussian and DOG pyramid, then conducting the SIFT algorithm to all 3 groups according to the priority level for image registration. Figure 5 presents the workflow of the proposed adaptive uniform registration algorithm.

To reduce to data processing volume, the SIFT registration is applied to the first 3 layers' DOG generated from the originalsized images. For the subsets which do not contain enough matching points, gradually enhancing the use ratio of the Gauss pyramid and the DOG images till the requirements of the matching points' amount is fulfilled or all 3 groups' Gauss pyramid SIFT algorithm processing finished.

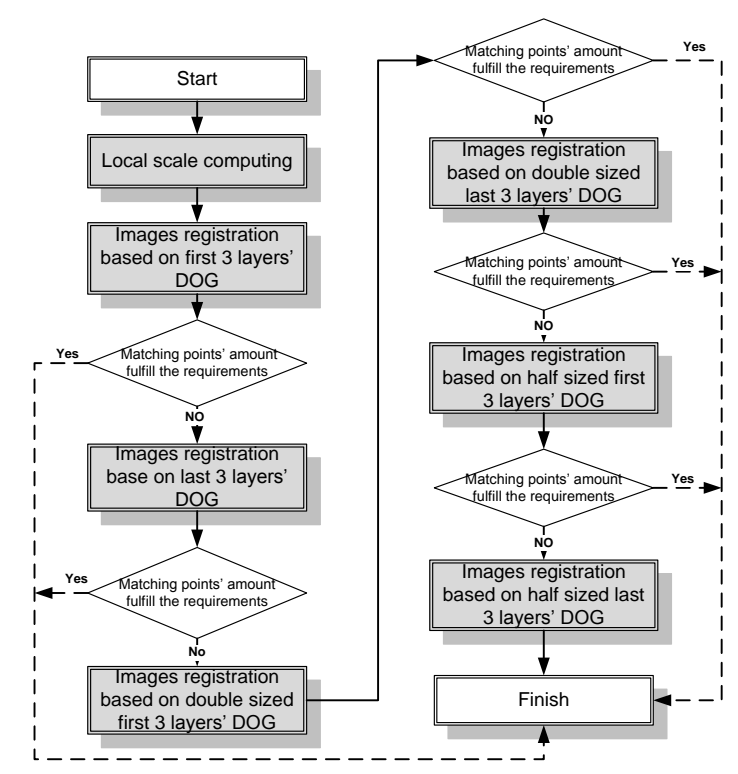

Figure 4 Workflow of the adaptive uniform registration algorithm

For the local area, the registration operation continues till one of the following requirement is fulfilled: (1) the required amount of matching points is achieved, or (2) the SIFT algorithm operation has been conducted to all 3 groups of Gauss pyramid. In addition, the number of required matches is controlled by the 
minimum and the maximum of the matching points: the registration process terminates if the matches exceed the maximum, while the process continues if the correct matches have not fulfill the minimum requirements.

\section{EXPERIMENT RESULTS AND ANALYSIS}

\subsection{Experiment results}

To ensure the versatility and effectiveness of the proposed algorithm, 6 pairs of CE-1 images, numbered 0192, 0232, 0397, 0488, 0522, 0621, were selected randomly in this research. Each pair contains both $\mathrm{B}$ and $\mathrm{F}$ view images. The first two pairs have the size of $512 \times 35672$ while the other four pairs are in the form of $512 \times 36360$. Based on the original SFIT algorithm and the proposed parallel and adaptive uniformdistributed registration method, 4 sets of experiments are conducted on the randomly selected images in this research. In the experimental process, the overlapping area is partitioned into several blocks first, then the parallel registration is carried out among the generated units based on the one-threaded, dualthreaded, tri-threaded and four-threaded process separately. The results of the time and speedup sheet of six groups images' parallel and the uniform-distributed registration experiment is presented in Table 1, and Figure 5 shows the statistic result of the speedup of the parallel registration.

Table 1 Time and speedup sheet of images parallel and the uniform-distributed registration experiment

\begin{tabular}{|ccrcrcrc|}
\hline \multirow{2}{*}{ Algorithm } & & \multicolumn{1}{c}{ Image 0192 } & \multicolumn{2}{c|}{ Image 0232 } & \multicolumn{2}{c|}{ Image 0397 } \\
\cline { 3 - 8 } & & $\begin{array}{c}\text { Time } \\
\text { (s) }\end{array}$ & $\begin{array}{c}\text { Spee } \\
\text { dup }\end{array}$ & $\begin{array}{c}\text { Time } \\
\text { (s) }\end{array}$ & $\begin{array}{c}\text { Spee } \\
\text { dup }\end{array}$ & $\begin{array}{c}\text { Time } \\
(\mathrm{s})\end{array}$ & $\begin{array}{c}\text { Spee } \\
\text { dup }\end{array}$ \\
\hline Standard & $1^{*}$ & 2260 & 1.00 & 2255 & 1.00 & 2308 & 1.00 \\
SIFT & 2 & 1481 & 1.53 & 1487 & 1.52 & 1519 & 1.52 \\
algorithm & 3 & 1205 & 1.88 & 1218 & 1.85 & 1241 & 1.86 \\
& 4 & 790 & 2.86 & 785 & 2.87 & 786 & 2.94 \\
\hline Proposed & 1 & 948 & 1.00 & 1263 & 1.00 & 1276 & 1.00 \\
algorithm & 2 & 714 & 1.33 & 978 & 1.29 & 952 & 1.34 \\
(matching & 3 & 615 & 1.54 & 860 & 1.47 & 835 & 1.53 \\
points $\geq 15)$ & 4 & 488 & 1.94 & 678 & 1.86 & 672 & 1.90 \\
\hline Proposed & 1 & 953 & 1.00 & 1274 & 1.00 & 1288 & 1.00 \\
algorithm & 2 & 729 & 1.31 & 985 & 1.29 & 951 & 1.35 \\
(matching & 3 & 621 & 1.53 & 868 & 1.47 & 827 & 1.56 \\
points $\in$ & & & & & & & \\
[15,30]) & 4 & 491 & 1.94 & 685 & 1.86 & 674 & 1.91 \\
\hline Proposed & 1 & 1167 & 1.00 & 1587 & 1.00 & 1563 & 1.00 \\
algorithm & 2 & 883 & 1.32 & 1216 & 1.31 & 1157 & 1.35 \\
(matching & 3 & 773 & 1.51 & 1046 & 1.52 & 994 & 1.57 \\
points=20) & 4 & 638 & 1.83 & 824 & 1.93 & 793 & 1.97 \\
\hline
\end{tabular}

\begin{tabular}{|c|c|c|c|c|c|c|c|}
\hline \multirow[b]{2}{*}{ Algorithm } & \multirow[b]{2}{*}{ Thread } & \multicolumn{2}{|c|}{ Image 0488} & \multicolumn{2}{|c|}{ Image 0522} & \multicolumn{2}{|c|}{ Image 0621} \\
\hline & & $\begin{array}{l}\text { Time } \\
\text { (s) }\end{array}$ & $\begin{array}{c}\text { Spee } \\
\text { dup }\end{array}$ & $\begin{array}{c}\text { Time } \\
\text { (s) }\end{array}$ & $\begin{array}{l}\text { Spee } \\
\text { dup }\end{array}$ & $\begin{array}{c}\text { Time } \\
(\mathrm{s})\end{array}$ & $\begin{array}{l}\text { Spee } \\
\text { dup }\end{array}$ \\
\hline \multirow{4}{*}{$\begin{array}{l}\text { Standard } \\
\text { SIFT } \\
\text { algorithm }\end{array}$} & $1 *$ & 2345 & 1.00 & 2310 & 1.00 & 2345 & 1.00 \\
\hline & 2 & 1531 & 1.53 & 1520 & 1.52 & 1545 & 1.52 \\
\hline & 3 & 1253 & 1.87 & 1228 & 1.88 & 1251 & 1.87 \\
\hline & 4 & 811 & 2.89 & 794 & 2.91 & 814 & 2.88 \\
\hline \multirow{4}{*}{$\begin{array}{l}\text { Proposed } \\
\text { algorithm } \\
\text { (matching } \\
\text { points } \geq 15 \text { ) }\end{array}$} & 1 & 1280 & 1.00 & 1341 & 1.00 & 1145 & 1.00 \\
\hline & 2 & 989 & 1.29 & 1040 & 1.29 & 876 & 1.31 \\
\hline & 3 & 833 & 1.54 & 900 & 1.49 & 783 & 1.46 \\
\hline & 4 & 674 & 1.90 & 725 & 1.85 & 612 & 1.87 \\
\hline \multirow{4}{*}{$\begin{array}{c}\text { Proposed } \\
\text { algorithm } \\
\text { (matching } \\
\text { points } \in[15 \text {, } \\
30] \text { ) }\end{array}$} & 1 & 1290 & 1.00 & 1352 & 1.00 & 1160 & 1.00 \\
\hline & 2 & 995 & 1.30 & 1053 & 1.28 & 898 & 1.29 \\
\hline & 3 & 840 & 1.54 & 906 & 1.49 & 785 & 1.48 \\
\hline & 4 & 683 & 1.89 & 735 & 1.84 & 605 & 1.92 \\
\hline \multirow{4}{*}{$\begin{array}{l}\text { Proposed } \\
\text { algorithm } \\
\text { (matching } \\
\text { points }=20 \text { ) }\end{array}$} & 1 & 1619 & 1.00 & 1659 & 1.00 & 1436 & 1.00 \\
\hline & 2 & 1217 & 1.33 & 1274 & 1.30 & 1079 & 1.33 \\
\hline & 3 & 1030 & 1.57 & 1080 & 1.54 & 944 & 1.52 \\
\hline & 4 & 825 & 1.96 & 853 & 1.94 & 726 & 1.98 \\
\hline
\end{tabular}

\section{* indicates the standard SIFT algorithm}

\subsection{Results Analysis}

In this section, the performance of a parallel and adaptive uniform-distributed registration method was presented and compared with the standard SIFT algorithm by using CE-1 lunar imageries as the experimental data. The experimental results are concluded as the followings:

1) By introducing the parallel processing and the uniformdistributed registration as two improvement factors, the efficiency of the CE-lunar imageries registration was dramatically improved. Since the goal of the proposed method in this research is to acquire satisfied number of the matching points rather than the quantity, and in the most circumstances the SIFT descriptor generating operation is only conducted on the Gaussian octaves and DoGs at local scale, the consumed time for the image registration is significantly reduced. Compared to the standard SIFT algorithm the uniformdistributed method could reduce the operation time by $29.3 \%$ $79.2 \%$, the average of which is $56.2 \%$. On the other hand, the parallel processing is also proved to be conductive to improve the registration efficiency. As can be seen in Table 1 , the speedup of 6 pairs of images could reach from 1.31 to 2.89 under 2-4 threaded operation.

2) Under the condition of the same number of threads, the processing time for the proposed method is much less than the standard SIFT algorithm for CE-1 lunar imageries. However, the improvement extent of the speedup indicator for the paper proposed method is not as dramatic as the standard SIFT with threads number increases. This because the extra calculation for the matching points counting and control is introduced in the proposed method. In this experiment, the speedup ration of each parallel algorithm does not growth linearly with the number of threads increases, this result accords with the general regulation of the parallel computing.

3) By applying the parallel and adaptive uniform-distributed registration method, the matching points in each divided blocks will be extracted based on the required amount, and this operation terminates when the amount is reached. For the blocks in which the matching points could not achieve the required amount, the Gauss pyramid and the DOG images will then be introduced for the image registration, thus realizing the uniform distribution of the matching points across the whole image. The analysis results suggest that the matching points are more uniform distributed in condition of the maximum value is set. Moreover, setting the maximum and minimum as the same value could also improve the uniform distribution of matching points effectively. 

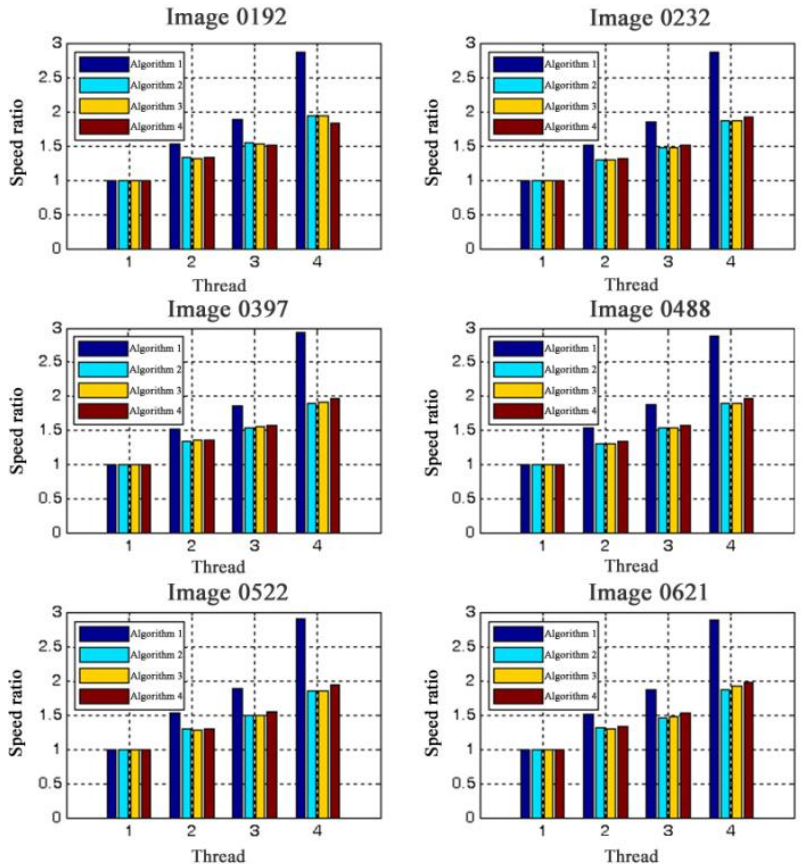

Figure 5 Speedup ration of parallel registration

\section{CONCLUSION}

Due to the dramatic change of the radiation information on the CE-1imageries, the traditional method based on the gray and line characters shows the limitation of achieving a satisfied result. Moreover, the registration process among lunar images which cover the whole moon has proved to be very timeconsuming. In this paper, based on the standard SIFT algorithm a parallel and adaptive uniform-distributed registration method for Chang'e-1 lunar remote sensed imagery was proposed. The experimental results show the applicability of the proposed method for CE-1 lunar imageries registration: it could generate the matching points uniformly and effectively, and thereby be used for the establishment of the lunar topographic map and lunar geodetic network. However, due to the limited experimental conditions, only the CE-1lunar imageries were applied for the registration. With the launch of the CE-2 and the other lunar satellites, the further researches should conduct higher multi-threads parallel processing experiment on large amount and multi-source data, and then establish a foundation for the automatic full-moon image matching and processing in the next step.

\section{REFERENCES}

David G. Lowe. Object Recognition from Local Scale-Invariant Features[C]// International Conference on Computer Vision. 1999: 1150-1157.

David G. Lowe. Distinctive Image Features from ScaleInvariant Keypoints[J]. International Journal of Computer Vision, 2004,60(2): 91-110.

Koenderink Jan J. The Structure of Images[J]. Biomedical Cybernetics, 1984,50(5):363-370.

Lindeberg T. Scale-Space Theory: a Basic Tool for Analysing Structures at Difference Scales[J]. Journal of Applied Statistics, 1994,21(2): 224-270.
J., GUO L. \& QIAN Z., et al. Determination of the Controlled Landing Trajectory of Chang'E-1 Satellite and the Coordinate Analysis of the Landing Point on the Moon[J]. Chinese Sci Bull, 2010, 55(9):752-757.

LIU J. Extracting and Matching of SIFT Feature for Simulated Lunar Environment[J]. Aerospace Control and Application,2009,35(5): 48-60.

LIU J. Feature Extracting and Matching Algorithm for Lunar Image Based on Image Enhencement[J]. Computer Simulation,2010,27(5):27-30.

Mikolajczyk K., Schmid C. A Performance Evaluation of Local Descriptors[J]. IEEE Transactions on Pattern Analysis and Machine Intelligence, 2005, 27(10):1615-1630.

SUN J., XU Z. A Review on Scale Space Method in Computer Vision[J].Chinese Journal of Engineering Mathematics,2005,22(6):951-962.

WANG R., WANG X. \& WANG J., et al. The Interior Accuracy Estimation in the Photogrammetric Calculation of the Stereoscopic Imagery of The Chang'e-1[J].Science of Surveying and Mapping,2008,33(2):5-7.

ZHAO B., YANG J.\& WEN D., et al. Design and On-orbit Measurement of Chang'e-1 Satellite CCD Stereo Camera[J]. Spacecraft Engineering,2009,18(1):30-36.

ZHAO B., YANG J. \& HE Y., et al. Lunar Exploration Optics [J]. Act a Photonic Sinica,2009,38(3):461-467.

ZHENG Y., OUYANG Z. Chang'E-1 Lunar Orbit: China's First Step into Deep Space Exploration[J]. Science \& Technology Review, 2007,25(5): 47-52. 